\title{
Is electronic approval appropriate?
}

Sometimes, a matter that appears to be trivial can initiate major disagreements. That is what happened after a monthly meeting of the Great Eastern University IACUC. Dr. Lawrence Covelli opened the meeting by asking for a motion to approve the minutes of the previous month's meeting. A motion was made and seconded, but Lana Phillipe, the IACUC administrator, whispered to Covelli that he had miscounted and there were not enough members present to make a quorum. "No problem," said Covelli, "we'll wait for a quorum and approve the minutes later on." When the quorum was present, Covelli began what turned out to be a long meeting, and by the time he remembered to ask for a vote on the minutes, members already were drifting out of the room, and once again, there was no quorum. Unbothered, Covelli said he would just poll the members by e-mail and get the minutes approved that way. That statement initiated his disagreement with Phillipe.
Phillipe was adamant that polling the committee for a vote on the minutes was unacceptable to both the US Department of Agriculture/Animal Care and the National Institutes of Health/Office of Laboratory Animal Welfare. Covelli was equally adamant that polling was only unacceptable under specific conditions, such as voting on the suspension of an animal activity or full committee review of a protocol. Covelli defended polling in other instances by using the Designated Member Review process as an example. Initially, the IACUC office would poll committee members to see if any person requested a full committee review. Phillipe disagreed with the comparison, saying that federal regulations did not require a full committee meeting to make that initial decision, because doing so would largely defeat the purpose of a Designated Member Review. She then reminded Covelli that both the Animal Welfare Act Regulations ${ }^{1}$ and the Public Health Service Policy on
Humane Care and Use of Laboratory Animals $^{2}$ require the keeping of minutes of IACUC meetings and, therefore, any vote to approve, modify or disapprove the minutes should be made like any other IACUC vote: at a quorum of the full committee and not by polling members after the meeting ended. "Oh," said Covelli, now becoming sarcastic, "are you saying that I can't poll the committee to see if we should go ahead with an investigation of a complaint?" "That's right," countered Phillipe, "you cannot use polling as a means of getting an IACUC vote that is directly or indirectly mandated by federal regulations."

Who do you think is right: Covelli or Phillipe?

1. Animal Welfare Act Regulations, 9 CFR (Chapter 1).

2. Public Health Service. Policy on Humane Care and Use of Laboratory Animals (US Department of Health and Human Services, Washington, DC, 1986; amended 2002).

\section{RESPONSE}

\section{Assurance should dictate}

\section{Ellen Teneriello, RVT, LATG, MA}

Both the Animal Welfare Act Regulations (AWARs; section 2.35a-2.35f) ${ }^{1}$ and Public Health Service Policy on Humane Care and Use of Laboratory Animals (PHS Policy; section IV.E) $)^{2}$ require institutions that use animals in research to keep certain types of records, including meeting minutes, attendance records, committee activities and deliberations, IACUC proposals and activities, semiannual reports and records of accrediting body determinations. The AWARs and PHS Policy do not include guidance on how the records should be created, but do specify that records should be kept on file for at least 3 years. Additionally, the PHS Policy (section IV. A) $)^{2}$ requires institutions who receive PHS funding to file an institutional Assurance with the Office of Laboratory Animal Welfare (OLAW), which should "fully describe the institution's program" for compliance with the PHS Policy, including "the procedures which the IACUC will follow to fulfill the requirements set forth in this Policy"2. Similarly, each institution is left to establish its own procedures for voting on approval or disapproval of records such as meeting minutes; these should also be included in the Assurance.

The concern for Great Eastern University is the issue of polling. Representatives of OLAW wrote, "Polling is defined as sequential, one on one communication, either in person or via telephone, email, fax,
US mail or by other similar means. Polling is an appropriate mechanism for providing all committee members with the opportunity to call for full review of protocol review"3. They went on to indicate that polling does not satisfy the definition of a convened quorum ${ }^{3}$. Therefore, it is important that Great Eastern University's Assurance document includes a description about various forms of communication established and used by the IACUC, such as e-mail voting.

In my opinion, Covelli can call for an e-mail vote on the minutes as long as the e-mail is distributed to the entire IACUC and the records of that vote include any objections or minority views.

It is important to note that voting by e-mail cannot substitute for a convened meeting when it comes to matters such as a vote on animal use activity protocols. In 